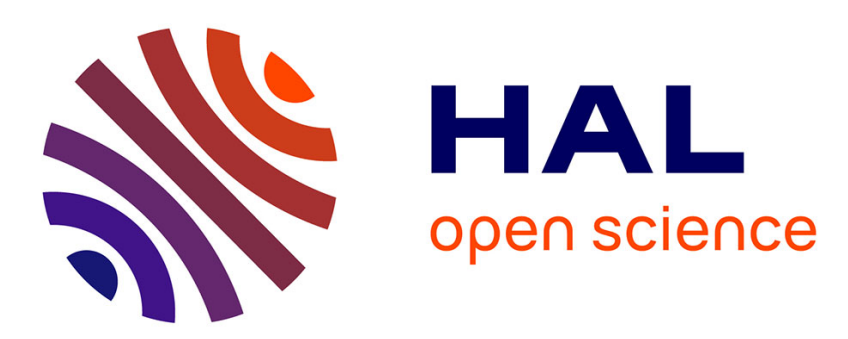

\title{
Extinction and coming down from infinity of continuous-state branching processes with competition in a Lévy environment
}

Hélène Leman, Juan Carlos Pardo Millan

\section{- To cite this version:}

Hélène Leman, Juan Carlos Pardo Millan. Extinction and coming down from infinity of continuousstate branching processes with competition in a Lévy environment. Journal of Applied Probability, 2021, 58 (1), pp.128 - 139. 10.1017/jpr.2020.77 . hal-01694581v3

\section{HAL Id: hal-01694581 \\ https://hal.science/hal-01694581v3}

Submitted on 4 Oct 2019

HAL is a multi-disciplinary open access archive for the deposit and dissemination of scientific research documents, whether they are published or not. The documents may come from teaching and research institutions in France or abroad, or from public or private research centers.
L'archive ouverte pluridisciplinaire HAL, est destinée au dépôt et à la diffusion de documents scientifiques de niveau recherche, publiés ou non, émanant des établissements d'enseignement et de recherche français ou étrangers, des laboratoires publics ou privés. 


\title{
Extinction and coming down from infinity of CB-processes with competition in a Lévy environment
}

\author{
H. Leman* and J.C. Pardo ${ }^{\dagger}$
}

June 4, 2019

\begin{abstract}
In this note, we are interested on the event of extinction and the property of coming down from infinity of continuous state branching (or CB for short) processes with competition in a Lévy environment whose branching mechanism satisfies the so-called Grey's condition. In particular, we deduce, under the assumption that the Lévy environment does not drift towards infinity, that for any starting point the process becomes extinct in finite time a.s. Moreover if we impose an integrability condition on the competition mechanism, then the process comes down from infinity regardless the long term behaviour of the environment.
\end{abstract}

KEY WORDS AND PHRASES: Continuous state branching processes in random environment, competition, extinction, coming down from infinity.

MSC 2000 subject classifications: 60J80, 60J75, 60J85.

\section{Introduction and main results.}

A continuous state branching process (or CB-process for short) is a $[0, \infty]$-valued strong Markov process $Y=\left(Y_{t}, t \geq 0\right)$ with cádlág paths satisfying the branching property, that is to say, for all $\theta \geq 0$ and $x, y \geq 0$,

$$
\mathbb{E}_{x+y}\left[e^{-\theta Y_{t}}\right]=\mathbb{E}_{x}\left[e^{-\theta Y_{t}}\right] \mathbb{E}_{y}\left[e^{-\theta Y_{t}}\right] .
$$

This model arises as the scaling limit of Bienaymé-Galton-Watson (or BGW for short) processes; where individuals behave independently one from each other and each individual gives birth to a random number of offspring, with the same offspring distribution (see for instance Grimvall [13]). Moreover, its law is completely characterized by the latter identity, i.e.

$$
\mathbb{E}_{x}\left[e^{-\theta Y_{t}}\right]=e^{-x u_{t}(\lambda)}, \quad t \geq 0,
$$

where $u$ is a differentiable function in $t$ satisfying

$$
\frac{\partial u_{t}(\lambda)}{\partial t}=-\psi\left(u_{t}(\lambda)\right), \quad u_{0}(\lambda)=\lambda,
$$

*Centro de Investigación en Matemáticas A.C. Calle Jalisco s/n. 36240 Guanajuato, México. E-mail: helene.leman@cimat.mx. Corresponding author

${ }^{\dagger}$ Centro de Investigación en Matemáticas A.C. Calle Jalisco s/n. 36240 Guanajuato, México. E-mail: jcpardo@cimat.mx 
and $\psi$ satisfies

$$
\psi(\lambda)=-b \lambda+\gamma^{2} \lambda^{2}+\int_{(0, \infty)}\left(e^{-\lambda x}-1+\lambda x\right) \mu(\mathrm{d} x), \quad \lambda \geq 0,
$$

where $a, \gamma \in \mathbb{R}$ and $\mu$ is a measure concentrated on $(0, \infty)$ such that

$$
\int_{(0, \infty)}\left(z \wedge z^{2}\right) \mu(\mathrm{d} z)<\infty
$$

The function $\psi$ is known as the branching mechanism of $Y$. A process in this class can also be defined as the unique non-negative strong solution of the following stochastic differential equation (SDE for short)

$$
Y_{t}=Y_{0}+b \int_{0}^{t} Y_{s} \mathrm{~d} s+\int_{0}^{t} \sqrt{2 \gamma^{2} Y_{s}} \mathrm{~d} B_{s}+\int_{0}^{t} \int_{(0, \infty)} \int_{0}^{Y_{s-}} z \widetilde{N}(\mathrm{~d} s, \mathrm{~d} z, \mathrm{~d} u),
$$

where $B=\left(B_{t}, t \geq 0\right)$ is a standard Brownian motion, $N(\mathrm{~d} s, \mathrm{~d} z, \mathrm{~d} u)$ is a Poisson random measure independent of $B$, with intensity $\mathrm{d} s \mu(\mathrm{d} z) \mathrm{d} u$ and $\widetilde{N}$ is the compensated measure of $N$. Solutions for this type of SDE has been studied before, see for instance Dawson and Li [8] and Caballero et al. [7] and the references therein.

A natural way to extend and make this model more realistic is by considering competition pressure. Such type of models has been considered recently by several authors, under the name of CB-processes with competition; see for instance Ba and Pardoux [2], Berestycki et al. [5], Foucart [11], Lambert [18], Ma [25] and Pardoux [28] and the references therein. A well known example of this family of processes is the so called logistic Feller diffusion which can be constructed as scaling limits of BGW-processes with competition, see for instance Lambert [18].

CB-processes with competition can also be defined as the unique strong solution of an SDE and they are determined by two components; a branching mechanism $\psi$ and a competition mechanism $g$. The competition mechanism $g$ is a non-decreasing continuous function on $[0, \infty)$ with $g(0)=0$. According to Ma [25] (see also Berestycki et al. [5]) a CB-process with competition $Y=\left(Y_{t}, t \geq 0\right.$ ) can be defined as the unique strong solution of the following SDE

$$
Y_{t}=Y_{0}+b \int_{0}^{t} Y_{s} \mathrm{~d} s-\int_{0}^{t} g\left(Y_{s}\right) \mathrm{d} s+\int_{0}^{t} \sqrt{2 \gamma^{2} Y_{s}} \mathrm{~d} B_{s}^{(b)}+\int_{0}^{t} \int_{(0, \infty)} \int_{0}^{Y_{s-}} z \widetilde{N}^{(b)}(\mathrm{d} s, \mathrm{~d} z, \mathrm{~d} u),
$$

where $B^{(b)}$ is a standard Brownian motion and $N^{(b)}$ is a Poisson random measure which is defined on $\mathbb{R}_{+}^{3}$, with intensity measure $\mathrm{d} s \mu(\mathrm{d} z) \mathrm{d} u$ such that $(1.3)$ is satisfied, and $\widetilde{N}^{(b)}$ denotes its compensated version.

Lambert [18] studied the long term behaviour of the logistic case i.e. $g(x)=c x^{2}$, for $x \geq 0$ and $c>0$, using a Lamperti-type representation (random time change) where the driven process turns out to be a generalised Ornstein-Uhlenbeck process driven by a spectrally positive Lévy process, here denoted by $X$, satisfying a logarithmic moment condition. To be more precise when the process $X$ is a subordinator then the associated logistic branching process $Y$ may converge to a specified distribution or to 0 in probability (see Theorem 3.4 in [18]). When $X$ is not a subordinator, then the process $Y$ goes to 0 a.s. Moreover, the process $Y$ gets extinct in finite time a.s. accordingly as

$$
\int^{\infty} \frac{\mathrm{d} u}{\psi(u)}<\infty
$$

which is the so-called Grey's condition. In [18], under Grey's condition, the Laplace transform of the extinction time was computed explicitly and the law of the process coming down from infinity was also determined. 
More general competition mechanisms were considered by $\mathrm{Ba}$ and Pardoux [2] in the case where the branching mechanism is of the form $\psi(u)=\gamma^{2} u^{2}$, for $u \geq 0$, see also Chapter 8 in the book of Pardoux [28]. Actually, they allow the competition mechanism $g$ to be a continuous function and not necessarily monotone and provided a necessary and sufficient condition for the process to become extinct. In this setting, if $g$ is negative and non-increasing then the competition mechanism can be interpreted as cooperation in the sense of Gonzalez-Casanova et al. [12].

Branching processes in random environment (BPREs) were first introduced and studied by Smith and Wilkinson [30] and since then they have attracted considerable interest (see for instance [1, 6] and the references therein). BPREs are interesting since they are more realistic models compared with classical branching processes and, from the mathematical point of view, they have new properties such as another phase transition in the subcritical regime. Scaling limits in the finite variance case were conjectured by Keiding [16] who introduced Feller diffusions in random environment. This conjecture was proved by Kurtz [17] and more recently by Bansaye and Simatos [3] in more general cases. The continuous state version, with an environment driven by a Lévy process, was recently introduced independently by He et al. [14] and Palau and Pardo [27], as the unique strong solution of an SDE (see below) under the name of CB-processes in Lévy environment.

In this paper, we are interested in CB-processes with competition in a Lévy environment which are defined as the unique strong solution of the following SDE

$$
\begin{array}{rl}
Z_{t}=Z_{0}+b \int_{0}^{t} Z_{s} \mathrm{~d} s-\int_{0}^{t} & g\left(Z_{s}\right) \mathrm{d} s+\int_{0}^{t} \sqrt{2 \gamma^{2} Z_{s}} \mathrm{~d} B_{s}^{(b)} \\
& +\int_{0}^{t} \int_{(0, \infty)} \int_{0}^{Z_{s-}} z \widetilde{N}^{(b)}(\mathrm{d} s, \mathrm{~d} z, \mathrm{~d} u)+\int_{0}^{t} Z_{s-} \mathrm{d} S_{s},
\end{array}
$$

where $g$ is a non-decreasing continuous function on $[0, \infty)$ with $g(0)=0, B^{(b)}$ and $N^{(b)}$ are defined as before and $S$ is a Lévy process independent of $B^{(b)}$ and $N^{(b)}$ which can be written as follows

$$
S_{t}=\mathrm{d} t+\sigma B_{t}^{(e)}+\int_{0}^{t} \int_{(-1,1)^{c}}\left(e^{z}-1\right) N^{(e)}(\mathrm{d} s, \mathrm{~d} z)+\int_{0}^{t} \int_{(-1,1)}\left(e^{z}-1\right) \tilde{N}^{(e)}(\mathrm{d} s, \mathrm{~d} z),
$$

with $\mathrm{d} \in \mathbb{R}, \sigma \geq 0, B^{(e)}=\left(B_{t}^{(e)}, t \geq 0\right)$ is a standard Brownian motion and $N^{(e)}$ is a Poisson random measure taking values on $\mathbb{R}_{+} \times \mathbb{R}$ with intensity $\mathrm{d} s \pi(\mathrm{d} z)$ satisfying

$$
\int_{\mathbb{R} \backslash\{0\}}\left(1 \wedge z^{2}\right) \pi(\mathrm{d} z)<\infty
$$

In particular, our aim is to determine under which conditions such family of processes becomes extinct (with positive probability or almost surely) and comes down from infinity.

For our purposes, we also introduce the auxiliary Lévy process which is a modification of $S$,

$$
K_{t}=\mathbf{m} t+\sigma B_{t}^{(e)}+\int_{0}^{t} \int_{(-1,1)^{c}} z N^{(e)}(\mathrm{d} s, \mathrm{~d} z)+\int_{0}^{t} \int_{(-1,1)} z \widetilde{N}^{(e)}(\mathrm{d} s, \mathrm{~d} z), \quad t \geq 0,
$$

where

$$
\mathbf{m}=b+\mathrm{d}-\frac{\sigma^{2}}{2}-\int_{(-1,1)}\left(e^{z}-1-z\right) \pi(\mathrm{d} z)
$$

It is important to note that the drift term $\mathbf{m}$ of the process $K$ provides the interaction between the demographic and environmental parameters.

We denote by $\mathbb{P}_{x}$ the law of $Z$ starting from $x>0$, and we define by $T_{0}=\inf \left\{t \geq 0, Z_{t}=0\right\}$ the first hitting time to 0 of $Z$, with the convention that $\inf \{\emptyset\}=\infty$. In the case without competition i.e. $g \equiv 0$, 
He et al. [14] proved that the so-called Grey's condition (1.5) is necessary and sufficient for CB-processes in a Lévy environment to become extinct with positive probability (see Theorem 4.1 in [14]). Moreover, if the auxiliary process $K$ does not drift to $\infty$ or equivalently

$$
\liminf _{t \rightarrow \infty} K_{t}=-\infty
$$

and Grey's condition (1.5) holds, then its associated CB-process in a Lévy environment becomes extinct at finite time a.s., see Corollary 4.4 in [14]. It is important to note that when condition (1.9) is fullfilled, the associated CB-process in a Lévy environment is critical or subcritical.

We also point out that under the assumption (1.3), the CB-process in a Lévy environment does not explode. The proof of this claim follows exactly the same arguments of Proposition 1 in [26] where the authors consider the specific case of Brownian environment. Indeed, in their arguments the environment does not play any role, when assumption (1.3) is fulfilled the non-explosion only depends on the branching mechanism.

Our first result, which follows from a comparison criteria for CB-processes with competition in a Lévy environment (see Lemma 2.1 below), gives a necessary condition under which they become extinct. Before stating it, we introduce the CB-process in a Lévy environment $Z^{\sharp}=\left(Z_{t}^{\sharp}, t \geq 0\right)$ as the unique strong solution of (1.6) but with $g \equiv 0$. For simplicity, we denote its law starting from $x>0$ by $\mathbb{P}_{x}^{\sharp}$.

Proposition 1.1. Assume that the Lévy measure $\mu$ associated to the branching mechanism $\psi$ satisfies (1.3). For $y \geq x \geq 0$, we have that $\left(Z, \mathbb{P}_{x}\right)$ is stochastically dominated by $\left(Z, \mathbb{P}_{y}\right)$, i.e.

$$
\mathbb{P}\left(Z_{t}(x) \leq Z_{t}(y) \text { for all } t \geq 0\right)=1,
$$

where $Z(v)$ denotes $\left(Z, \mathbb{P}_{v}\right)$, under $\mathbb{P}$. Moreover, the process $\left(Z, \mathbb{P}_{x}\right)$ is stochastically dominated by $\left(Z^{\sharp}, \mathbb{P}_{y}^{\sharp}\right)$ and, in particular, if the branching mechanism $\psi$ satisfies Grey's condition (1.5), then $\left(Z, \mathbb{P}_{x}\right)$ becomes extinct with positive probability and its semigroup is strong Feller. Furthermore if $K$ does not drift to $\infty$ or equivalently satisfies $(1.9)$ then $\left(Z, \mathbb{P}_{x}\right)$ becomes extinct at finite time a.s.

For the sequel, we always assume that Grey's condition is fulfilled. In other words, the CB-processes with competition in a Lévy environment that we are considering here become extinct with positive probability and are strong Feller.

We now state our main result which provides a sufficient condition on the competition mechanism for CB-processes with competition in a Lévy environment to become extinct a.s. even for favorable environments, i.e. when $K$ drifts to $+\infty$. In other words, the condition on the competition parameter is so strong that the process become extinct regardless the long term behaviour of the environment. To this aim, we assume the following integral condition on the competition mechanism $g$ : assume that there exists $z_{0}>0$ such that $g\left(z_{0}\right)>0$ and

$$
\int_{z_{0}}^{\infty} \frac{\mathrm{d} y}{g(y)}<\infty
$$

Actually, the above condition implies that the associated CB-process with competition in a Lévy random environment comes down from infinity. This phenomenon has been observed and studied by several authors in branching processes with interactions, see for instance González-Casanova et al. [12], Lambert [18], Li [23], Li et al. [24] and Pardoux [28] and also for stable jump diffusions by Döring and Kyprianuo [9] and some jump diffusions by Bansaye [4]. Formally, we define the property of coming down from infinity in the sense that $\infty$ is a continuous entrance point, i.e.

$$
\lim _{M \rightarrow \infty} \lim _{x \rightarrow \infty} \mathbb{P}_{x}\left(T_{M}<t\right)=1 \quad \text { for all } t>0,
$$


where $T_{M}=\inf \left\{t \geq 0: Z_{t} \leq M\right\}$ and the original process can be extended into a Feller process on $[0, \infty]$ (see for instance Theorem 20.13 in Kallenberg [15] for the diffusion case or Definition 2.2 for Feller processes in [9]).

Theorem 1.2. Assume that the Lévy measure $\mu$ associated to the branching mechanism satisfies (1.3). If Grey's condition (1.5) and (1.10) hold, then

$$
\sup _{x>0} \mathbb{E}_{x}\left[T_{0}\right]<\infty
$$

the boundary point $\infty$ is a continuous entrance point and the process $Z$ comes down from infinity.

The previous result can be applied to the particular case when the competition mechanism is logistic (i.e. $g(x)=c x^{2}$ ) and the random environment is driven by a Brownian motion. Actually, further explicit computations can be carried out for the Laplace transform of the extinction time under $\mathbb{P}_{\infty}$, the law of the process starting from $\infty$, as it is done in Leman and Pardo [22] where the case of the logistic branching process in a Brownian environment is presented.

The remainder of this note is devoted to the proofs.

\section{Proofs}

In order to prove Proposition 1.1, we introduce the following stochastic processes as unique strong solutions of the SDE's. For $i=1,2$, we let

$$
Z_{t}^{(i)}=Z_{0}^{(i)}+\int_{0}^{t} g_{i}\left(Z_{s}^{(i)}\right) \mathrm{d} s+\int_{0}^{t} \sqrt{2 \gamma^{2} Z_{s}^{(i)}} \mathrm{d} B_{s}^{(b)}+\int_{0}^{t} \int_{(0, \infty)} \int_{0}^{Z_{s-}^{(i)}} z \widetilde{N}^{(b)}(\mathrm{d} s, \mathrm{~d} z, \mathrm{~d} u)+\int_{0}^{t} Z_{s-}^{(i)} \mathrm{d} S_{s}^{(i)},
$$

where

$$
S_{t}^{(i)}=\mathrm{d} t+\sigma B_{t}^{(e)}+\int_{0}^{t} \int_{(-1,1)^{c}} b_{i}(z) N^{(e)}(\mathrm{d} s, \mathrm{~d} z)+\int_{0}^{t} \int_{(-1,1)}\left(e^{z}-1\right) \tilde{N}^{(e)}(\mathrm{d} s, \mathrm{~d} z),
$$

with $g_{1}(z) \geq g_{2}(z)$, for $z \geq 0$, and $b_{1}(z) \geq b_{2}(z)$ for $z \in \mathbb{R}$ such that, for $i=1,2$

$$
b_{i}(z)+1 \geq 0, \quad \text { for } \quad z \in \mathbb{R} .
$$

We also assume that for each $m \geq 0$, there is a non-decreasing concave function $z \mapsto r_{m}(z)$ on $\mathbb{R}_{+}$ satisfying $\int_{0+} r_{m}(z) \mathrm{d} z=\infty$ and

$$
\left|g_{i}(x)-g_{i}(y)\right|+\mathrm{d}|x-y|+|x-y| \int_{(-1,1)^{c}}\left(\left|b_{i}(z)\right| \wedge m\right) \pi(\mathrm{d} z) \leq r_{m}(|x-y|), \quad \text { for } \quad i=1,2,
$$

for every $0 \leq x, y \leq m$. According to Proposition 1 in Palau and Pardo, the previous SDE's possess unique positive strong solutions that we denote by $Z^{(i)}$ for $i=1,2$.

Our next result can be deduced using similar arguments as those used in the proof of Theorem $2.2 \mathrm{in}$ [8]. For simplicity on exposition we provide its complete proof.

Lemma 2.1. If $Z_{0}^{(1)} \geq Z_{0}^{(2)}$, a.s. then

$$
\mathbb{P}\left(Z_{t}^{(2)} \leq Z_{t}^{(1)} \text { for all } t \geq 0\right)=1 .
$$


Proof. Let $\tau_{m}=\inf \left\{t \geq 0: Z_{s}^{(1)} \geq m\right.$ or $\left.Z_{s}^{(2)} \geq m\right\}$ for $m \geq 1$. According to the proof of Proposition 1 in Palau and Pardo [27], for $i=1,2$, we have $Z_{t}^{(i)}=Z_{t}^{(i, m)}$ for $t<\tau_{m}$, where $Z^{(i, m)}$ is the unique strong solution to

$$
\begin{aligned}
Z_{t}^{(i, m)} & =Z_{0}^{(i)}+\int_{0}^{t} g_{i}\left(Z_{s}^{(i, m)} \wedge m\right) \mathrm{d} s+\int_{0}^{t} \sqrt{2 \gamma^{2} Z_{s}^{(i, m)} \wedge m} \mathrm{~d} B_{s}^{(b)} \\
& +\int_{0}^{t} \int_{(0, \infty)} \int_{0}^{Z_{s-}^{(i, m)} \wedge m}(z \wedge m) \widetilde{N}^{(b)}(\mathrm{d} s, \mathrm{~d} z, \mathrm{~d} u)+\int_{0}^{t}\left(Z_{s-}^{(i, m)} \wedge m\right) \mathrm{d} S_{s}^{(i, m)},
\end{aligned}
$$

where

$$
S_{t}^{(i)}=\mathrm{d} t+\sigma B_{t}^{(e)}+\int_{0}^{t} \int_{(-1,1)^{c}}\left(b_{i}(z) \wedge m\right) N^{(e)}(\mathrm{d} s, \mathrm{~d} z)+\int_{0}^{t} \int_{(-1,1)}\left(\left(e^{z}-1\right) \wedge m\right) \tilde{N}^{(e)}(\mathrm{d} s, \mathrm{~d} z) .
$$

In other words for $m \geq 1$, we have

$$
\mathbb{P}\left(Z_{t}^{(1)} \geq Z_{t}^{(2)}, \text { for all } t<\tau_{m}\right)=\mathbb{P}\left(Z_{t}^{(1, m)} \geq Z_{t}^{(2, m)} \text {, for all } t<\tau_{m}\right) .
$$

Then, a direct application of Theorem 2.2 in [8] implies that the latter probability equals one. This ends the proof of Lemma 2.1.

Proof of Proposition 1.1. The first statement follows directly from Lemma 2.1 by taking

$$
g_{1}(z)=g_{2}(z)=(\mathrm{d}+b) z-g(z) \quad \text { for } z \geq 0 \quad \text { and } \quad b_{1}(z)=b_{2}(z)=e^{z}-1 \quad \text { for } z \in \mathbb{R} .
$$

For the second statement, we recall that the competition mechanism $g$ is positive and non-decreasing implying that we can take $g_{1}(z)=(\mathrm{d}+b) z, g_{2}(z)=(\mathrm{d}+b) z-g(z)$ and $b_{1}(z)=b_{2}(z)=e^{z}-1$. Again from Lemma 2.1 , we deduce that the process $\left(Z, \mathbb{P}_{x}\right)$ is stochastically dominated by $\left(Z^{\sharp}, \mathbb{P}_{y}^{\sharp}\right)$ for $y \geq x$. In other words, from Theorem 4.1 and Corollary 4.4 in [14], we deduce that $\left(Z, \mathbb{P}_{x}\right)$ becomes extinct with positive probability and that if $K$ does not drift to $\infty$ or equivalently satisfies (1.9) then the process becomes extinct at finite time a.s.

In order to conclude our proof, it remains to deduce that the process $\left(Z, \mathbb{P}_{x}\right)$ is strong Feller under Grey's condition (1.5). To do so, we use a similar argument as in Theorem 4.5 [14]. We introduce another formulation of CB-processes with competition in a Lévy environment for all initial values. From Theorem III.6 in El Karoui and Méléard [10], on an extension of the original probability space we can define $W(\mathrm{~d} s, \mathrm{~d} u)$ a time-space Gaussian white noise on $(0, \infty)^{2}$ with intensity $\mathrm{d} s \mathrm{~d} u$ such that (1.6) may be rewritten as follows

$$
\begin{aligned}
Z_{t}=x+b \int_{0}^{t} Z_{s} \mathrm{~d} s & -\int_{0}^{t} g\left(Z_{s}\right) \mathrm{d} s+\sqrt{2 \gamma^{2}} \int_{0}^{t} \int_{0}^{Z_{s}} W(\mathrm{~d} s, \mathrm{~d} u) \\
& +\int_{0}^{t} \int_{(0, \infty)} \int_{0}^{Z_{s-}} z \widetilde{N}^{(b)}(\mathrm{d} s, \mathrm{~d} z, \mathrm{~d} u)+\int_{0}^{t} Z_{s-} \mathrm{d} S_{s} .
\end{aligned}
$$

According to Proposition 1 in Palau and Pardo [27] (see also the proof of Theorem 4.5 of He et al. [14] for the case $g \equiv 0$ ), for each $x \geq 0$, there is a unique strong solution of the previous SDE that we denote by $\left(\Gamma_{t}^{(x)}, t \geq 0\right)$ with $\Gamma_{0}^{(x)}=x$, which is also a Markov process with the same transition semigroup as $\left(Z_{t}, \mathbb{P}_{x}\right)$, i.e.

$$
\mathrm{P}_{t} f(z):=\mathbb{E}_{z}\left[f\left(Z_{t}\right)\right]=\mathbb{E}\left[f\left(\Gamma_{t}^{(z)}\right)\right]
$$


Similar arguments as those used in the proof of Lemma 2.1 allow us to conclude that, for $y \geq 0$, we have

$$
\mathbb{P}\left(\Gamma_{t}^{(y)} \geq \Gamma_{t}^{(x)}, \quad \text { for all } t \geq 0\right)=1 .
$$

We claim that the process $\Gamma_{t}^{(x, y)}:=\Gamma_{t}^{(y)}-\Gamma_{t}^{(x)}$, for $t \geq 0$, satisfies a similar equation. Indeed

$$
\begin{gathered}
\Gamma_{t}^{(x, y)}:=y-x+b \int_{0}^{t} \Gamma_{s}^{(x, y)} \mathrm{d} s-\int_{0}^{t} G_{\Gamma_{t}^{(x)}}\left(\Gamma_{s}^{(x, y)}\right) \mathrm{d} s+\sqrt{2 \gamma^{2}} \int_{0}^{t} \int_{0}^{\Gamma_{s}^{(x, y)}} W^{\prime}(\mathrm{d} s, \mathrm{~d} u) \\
+\int_{0}^{t} \int_{(0, \infty)} \int_{0}^{\Gamma_{s-}^{(x, y)}} z \widetilde{N}^{(b) \prime}(\mathrm{d} s, \mathrm{~d} z, \mathrm{~d} u)+\int_{0}^{t} \Gamma_{s-}^{(x, y)} \mathrm{d} S_{s}
\end{gathered}
$$

where $G_{x}(z)=g(x+z)-g(x)$ is a non-decreasing continuous and positive function on $[0, \infty)$,

$$
W^{\prime}(\mathrm{d} s, \mathrm{~d} u)=W\left(\mathrm{~d} s, \mathrm{~d} u+\Gamma_{s}^{(x)}\right),
$$

and

$$
\widetilde{N}_{x}^{(b) \prime}(\mathrm{d} s, \mathrm{~d} z, \mathrm{~d} u)=\widetilde{N}_{x}^{(b)}\left(\mathrm{d} s, \mathrm{~d} z, \mathrm{~d} u+\Gamma_{s}^{(x)}\right)
$$

which are respectively a Gaussian white noise with intensity $\mathrm{d} s \mathrm{~d} u$ and a Poisson random measure with intensity $\mathrm{d} s \mu(\mathrm{d} z) \mathrm{d} u$. Using again the version of Lemma 2.1 for the SDE with Gaussian white noise, we deduce

$$
\mathbb{P}\left(\Gamma_{t}^{(x, y), \sharp} \geq \Gamma_{t}^{(x, y)}, \quad \text { for all } \quad t \geq 0\right)=1,
$$

where the process $\Gamma^{(x, y), \sharp}$ is the unique strong solution of

$$
\begin{aligned}
\Gamma_{t}^{(x, y), \sharp}:=y-x+b \int_{0}^{t} \Gamma_{s}^{(x, y), \sharp} \mathrm{d} s+\sqrt{2 \gamma^{2}} \int_{0}^{t} \int_{0}^{\Gamma_{s}^{(x, y), \sharp}} W^{\prime}(\mathrm{d} s, \mathrm{~d} u) \\
\quad+\int_{0}^{t} \int_{(0, \infty)} \int_{0}^{\Gamma^{(x, y), \sharp}} z \widetilde{N}^{(b) \prime}(\mathrm{d} s, \mathrm{~d} z, \mathrm{~d} u)+\int_{0}^{t} \Gamma_{s-}^{(x, y), \sharp} \mathrm{d} S_{s},
\end{aligned}
$$

which is equivalent to $\left(Z^{\sharp}, \mathbb{P}_{y-x}^{\sharp}\right)$. In other words, the process $\Gamma^{(x, y)}$ hits 0 a.s., and from its dynamics we observe that 0 is an absorbing boundary.

Now, let $f \in \mathcal{B}_{b}(\mathbb{R})$, the space of bounded measurable functions, and let

$$
T^{(x, y)}=\inf \left\{t \geq 0: \Gamma_{t}^{(y)}=\Gamma_{t}^{(x)}\right\} .
$$

Then $\Gamma_{t}^{(y)}=\Gamma_{t}^{(x)}$ for $t \geq T^{(x, y)}$ and observe,

$$
\begin{aligned}
\left|\mathrm{P}_{t} f(y)-\mathrm{P}_{t} f(x)\right| & \leq \mathbb{E}\left[\left|f\left(\Gamma_{t}^{(y)}\right)-f\left(\Gamma_{t}^{(x)}\right)\right| \mathbf{1}_{\left\{t<T^{(x, y)}\right\}}\right] \\
& \leq 2\|f\|_{\infty} \mathbb{P}\left(t<T^{(x, y)}\right) \\
& \leq 2\|f\|_{\infty} \mathbb{P}_{y-x}^{\sharp}\left(T_{0}>t\right) \\
& \leq 2\|f\|_{\infty} \mathbb{E}\left[1-e^{-(y-x) v_{t}(0, \infty, \bar{K})}\right],
\end{aligned}
$$

where $\|\cdot\|_{\infty}$ denotes the supremum norm and $v_{t}(0, \infty, \bar{K})$ is a functional of the environment such that

$$
\mathbb{P}_{y}^{\sharp}\left(Z_{t}=0 \mid \bar{K}\right)=e^{-y v_{t}(0, \infty, \bar{K})}>0,
$$

see for instance Theorems 4.1 and 4.3 in He et al. [14]. In other words, $\left|\mathrm{P}_{t} f(y)-\mathrm{P}_{t} f(x)\right|$ goes to 0 as $|x-y|$ goes to 0 , implying that $\mathrm{P}_{t} f$ is a continuous function on $[0, \infty)$. The proof is now complete. 
We now prove Theorem 1.2.

Proof of Theorem 1.2. First of all, from Lemma 2.1, it is enough to prove our result for a process with a random environment which has no downward jumps larger than $1-e^{-1}$. Hence, we assume in all this proof that $Z$ is solution to (1.6) with

$$
S_{t}=\mathrm{d} t+\sigma B_{t}^{(e)}+\int_{0}^{t} \int_{(1, \infty)}\left(e^{z}-1\right) N^{(e)}(\mathrm{d} s, \mathrm{~d} z)+\int_{0}^{t} \int_{(-1,1)}\left(e^{z}-1\right) \widetilde{N}^{(e)}(\mathrm{d} s, \mathrm{~d} z),
$$

Our first step is to prove that the expectation of the extinction time of the process is finite. Recall that $T_{M}$ denotes the first passage time for the process $Z$ below a level $M>0$, i.e. $T_{M}:=\inf \left\{t \geq 0, Z_{t} \leq M\right\}$. As we will see below, the finiteness of the first moment of such random times will be useful for deducing our result. Hence, we first show that there exist $M>0$ such that

$$
\sup _{x \geq 0} \mathbb{E}_{x}\left[T_{M}\right]=\sup _{x \geq M} \mathbb{E}_{x}\left[T_{M}\right]<\infty
$$

In order to deduce (2.3), we use similar arguments as those used in Le [19]. With this goal in mind, we observe from Assumption (1.10) that

$$
\lim _{y \rightarrow+\infty} \frac{g(y)-\theta y}{y}=\infty
$$

for $\theta:=\max \{b+\mathrm{d}, 0\}$. In addition from Lemma 2.3 in Le and Pardoux [20], we deduce that there exists $a_{0}>0$ such that $g(y)-\theta y>0$ for any $y \geq a_{0}$ and

$$
\int_{a_{0}}^{\infty} \frac{\mathrm{d} y}{g(y)-\theta y}<\infty
$$

We then introduce $A>\theta(e-1)$ large enough such that the inequality below holds

$$
\begin{aligned}
C(A):=1-\left(\frac{\theta\left(2 \gamma^{2}+\sigma^{2}\right)}{2 A^{2}}+\frac{\theta}{A(A-\theta)} \int_{(0,1)} z^{2} \mu(\mathrm{d} z)+\frac{1}{A}\left(\int_{(1, \infty)} z \mu(\mathrm{d} z)+\bar{\pi}(1)\right)\right. \\
\left.+\left(\frac{\theta}{A^{2}}+\frac{\theta}{A\left(A-\theta\left(e^{1}-1\right)\right)}\right) \int_{(-1,1)} z^{2} \pi(\mathrm{d} z)\right)>0
\end{aligned}
$$

where $\bar{\pi}(x)=\pi((x, \infty)), x \geq 0$. From (2.4) and (2.5), it is clear that there exists a constant $M>\left(a_{0}+1\right) e$ such that

$$
\int_{M e^{-1}}^{\infty} \frac{\mathrm{d} w}{g(w)-\theta w} \leq \frac{1}{A} \quad \text { and } \quad g(y)-\theta y \geq A y \geq A, \quad \text { for all } \quad y \geq M e^{-1}
$$

Such constant $M$ will be our threshold. For our purposes, we define the function $G$ in $C^{2}(\mathbb{R})$ as follows

$$
G(y)= \begin{cases}\int_{a_{0}}^{y} \frac{\mathrm{d} w}{g(w)-\theta w} & \text { if } y \geq a_{0}+1 \\ 0 & \text { if } y \leq a_{0}\end{cases}
$$


and such that $G$ is non-negative and non-decreasing. Thus applying Itô's formula to $G\left(Z_{t \wedge T_{M}}\right)$, we find

$$
\begin{aligned}
G\left(Z_{t \wedge T_{M}}\right)-G\left(Z_{0}\right) & =-t \wedge T_{M}-\int_{0}^{t \wedge T_{M}} \frac{g^{\prime}\left(Z_{s}\right)-\theta}{\left(g\left(Z_{s}\right)-\theta Z_{s}\right)^{2}}\left(\gamma^{2} Z_{s}+\frac{\sigma^{2}}{2} Z_{s}^{2}\right) \mathrm{d} s \\
& +\int_{0}^{t \wedge T_{M}} \frac{\sqrt{2 \gamma^{2} Z_{s}}}{g\left(Z_{s}\right)-\theta Z_{s}} \mathrm{~d} B_{s}^{(b)}+\int_{0}^{t \wedge T_{M}} \frac{\sigma Z_{s}}{g\left(Z_{s}\right)-\theta Z_{s}} \mathrm{~d} B_{s}^{(e)} \\
& +\int_{0}^{t \wedge T_{M}} \int_{(0, \infty)} Z_{s}\left(G\left(Z_{s}+z\right)-G\left(Z_{s}\right)-\frac{z}{g\left(Z_{s}\right)-\theta Z_{s}}\right) \mu(\mathrm{d} z) \mathrm{d} s \\
& +\int_{0}^{t \wedge T_{M}} \int_{(0, \infty)} \int_{0}^{Z_{s-}}\left[G\left(Z_{s-}+z\right)-G\left(Z_{s-}\right)\right] \tilde{N}^{(b)}(\mathrm{d} s, \mathrm{~d} z, \mathrm{~d} u) \\
& +\int_{0}^{t \wedge T_{M}} \int_{(1, \infty)}\left[G\left(e^{z} Z_{s-}\right)-G\left(Z_{s-}\right)\right] N^{(e)}(\mathrm{d} s, \mathrm{~d} z) \\
& +\int_{0}^{t \wedge T_{M}} \int_{(-1,1)}\left[G\left(e^{z} Z_{s-}\right)-G\left(Z_{s-}\right)\right] \tilde{N}^{(e)}(\mathrm{d} s, \mathrm{~d} z) \\
& +\int_{0}^{t \wedge T_{M}} \int_{(-1,1)}\left(G\left(e^{z} Z_{s}\right)-G\left(Z_{s}\right)-\frac{\left(e^{z}-1\right) Z_{s}}{g\left(Z_{s}\right)-\theta Z_{s}}\right) \pi(\mathrm{d} z) \mathrm{d} s .
\end{aligned}
$$

Firstly, note that $Z_{s} \geq M e^{-1}>a_{0}+1$ for any $s \leq t \wedge T_{M}$, in other words, we have an explicit formula for $G\left(Z_{s}\right)$. Next, we take expectations under the assumption that the process $Z$ starts at $x \geq M$, in both sides of the previous identity and we study separately each term of the right-hand side. Our aim is to show that each expectation can be bounded from above using $\mathbb{E}_{x}\left[t \wedge T_{M}\right]$. For simplicity, we enumerate the lines in order of appearance.

(1) For the first integral of the right hand side of (2.8), we recall that $Z_{s} \geq M e^{-1}$ for $s \leq t \wedge T_{M}$, that $g$ is non-decreasing and we use the second formula in (2.7) to deduce

$$
\mathbb{E}_{x}\left[\int_{0}^{t \wedge T_{M}} \frac{\theta-g^{\prime}\left(Z_{s}\right)}{\left(g\left(Z_{s}\right)-\theta Z_{s}\right)^{2}}\left(\gamma^{2} Z_{s}+\frac{\sigma^{2}}{2} Z_{s}^{2}\right) \mathrm{d} s\right] \leq \frac{\theta\left(2 \gamma^{2}+\sigma^{2}\right)}{2 A^{2}} \mathbb{E}_{x}\left[t \wedge T_{M}\right] .
$$

(2) Studying the quadratic variation of both continuous local martingales of the second line of the right-hand side of (2.8) together with the second formula in (2.7), we observe that both processes are real martingales. Therefore their expectations are equal to 0.

(3) We study the integral that appears in the third line in $(2.8)$ by separating $(0, \infty)$ into two parts $(0,1]$ and $(1, \infty)$. We first deal with the integral restricted to $(0,1)$. Since $g$ is non-decreasing, we bound $G\left(Z_{s}+z\right)-G\left(Z_{s}\right)$ from above by $z\left(g\left(Z_{s}\right)-\theta\left(Z_{s}+z\right)\right)^{-1}$. In addition with the second formula in $(2.7)$, we obtain the following upper bound

$$
\begin{aligned}
\mathbb{E}_{x}\left[\int_{0}^{t \wedge T_{M}} \int_{(0,1)} Z_{s}\left[G\left(Z_{s}+z\right)-G\left(Z_{s}\right)-\frac{z}{g\left(Z_{s}\right)-\theta Z_{s}}\right] \mu(\mathrm{d} z) \mathrm{d} s\right] \\
\leq \mathbb{E}_{x}\left[\int_{0}^{t \wedge T_{M}} \int_{(0,1)} \frac{\theta z^{2} Z_{s}}{\left(g\left(Z_{s}\right)-\theta\left(Z_{s}+z\right)\right)\left(g\left(Z_{s}\right)-\theta Z_{s}\right)} \mu(\mathrm{d} z) \mathrm{d} s\right] \\
\leq \mathbb{E}_{x}\left[t \wedge T_{M}\right] \frac{\theta}{A(A-\theta)} \int_{(0,1)} z^{2} \mu(\mathrm{d} z)<\infty .
\end{aligned}
$$

Concerning the integral restricted to $(1, \infty)$, we drop the last term, which is negative, and we use again 
the second formula in (2.7) to bound $G\left(Z_{s}+z\right)-G\left(Z_{s}\right)$ and find

$$
\begin{aligned}
\mathbb{E}_{x}\left[\int_{0}^{t \wedge T_{M}} \int_{(1, \infty)} Z_{s}\left[G\left(Z_{s}+z\right)-G\left(Z_{s}\right)-\frac{z}{g\left(Z_{s}\right)-\theta Z_{s}}\right] \mu(\mathrm{d} z) \mathrm{d} s\right] \\
\leq \mathbb{E}_{x}\left[\int_{0}^{t \wedge T_{M}} \int_{(1, \infty)} \int_{0}^{z} \frac{Z_{s}}{A\left(Z_{s}+w\right)} \mathrm{d} w \mu(\mathrm{d} z) \mathrm{d} s\right] \leq \frac{\int_{(1, \infty)} z \mu(\mathrm{d} z)}{A} \mathbb{E}_{x}\left[t \wedge T_{M}\right] .
\end{aligned}
$$

(4) Again, we split the interval $(0, \infty)$ into $(0,1]$ and $(1, \infty)$ and use similar computations as in part (3) in order to deduce that the integral restricted to $(0,1]$ is a square integrable martingale and the integral restricted to $(1, \infty)$ is a martingale. In other words, we manipulate

$$
\mathbb{E}_{x}\left[\int_{0}^{t \wedge T_{M}} \int_{(0, \infty)} Z_{s} f\left(G\left(Z_{s}+z\right)-G\left(Z_{s}\right), z\right) \mu(\mathrm{d} z) \mathrm{d} s\right],
$$

with $f(x, z)=x^{2} \mathbf{1}_{(0,1]}(z)$ and $f(x, z)=|x| \mathbf{1}_{(1, \infty)}(z)$ respectively. Their expectations are thus 0.

(5) Similarly, using Fubini's Theorem and the first inequality in (2.7), we deduce

$$
\begin{aligned}
\mathbb{E}_{x}\left[\int_{0}^{t \wedge T_{M}} \int_{(1, \infty)}\left|G\left(e^{z} Z_{s-}\right)-G\left(Z_{s-}\right)\right| \pi(\mathrm{d} z) \mathrm{d} s\right] & \leq \mathbb{E}_{x}\left[\int_{0}^{t \wedge T_{M}} \int_{Z_{s}}^{\infty} \frac{\mathrm{d} w}{g(w)-\theta w}\left(\int_{1}^{\infty} \pi(\mathrm{d} z)\right) \mathrm{d} s\right] \\
& \leq t \bar{\pi}(1)\left(\int_{M e^{-1}}^{\infty} \frac{\mathrm{d} w}{g(w)-\theta w}\right) \leq \frac{t}{A} \bar{\pi}(1) .
\end{aligned}
$$

In other words, the stochastic integral of the fifth term can be written as the sum of a martingale and a finite variation process. Moreover its expectation is bounded from above by $\mathbb{E}\left[t \wedge T_{M}\right] \bar{\pi}(1) / A$.

(6) Since $g(w)-\theta w \geq A w$, we find that the integral term of the sixth line is a square integrable martingale. Indeed, we observe

$$
\begin{aligned}
\mathbb{E}\left[\int_{0}^{t \wedge T_{M}} \int_{(-1,1)}\left(\int_{Z_{s}}^{e^{z} Z_{s}} \frac{\mathrm{d} w}{g(w)-\theta w}\right)^{2} \pi(\mathrm{d} z) \mathrm{d} s\right] & \leq \mathbb{E}\left[\int_{0}^{t \wedge T_{M}} \int_{(-1,1)}\left(\int_{Z_{s}}^{e^{z} Z_{s}} \frac{\mathrm{d} w}{A w}\right)^{2} \pi(\mathrm{d} z) \mathrm{d} s\right] \\
& \leq \frac{t}{A^{2}} \int_{(-1,1)} z^{2} \pi(\mathrm{d} z)<\infty
\end{aligned}
$$

In other words, its expectation is equal to 0 .

(7) Finally, we study the last line in (2.8) by splitting again the integral into two parts, i.e. we split $(-1,1)$ into $(-1,0]$ and $(0,1)$. Thus, using again the second inequality of $(2.7)$ and the fact that $A>\theta(e-1)$, we deduce that for any $w \in\left[0, y\left(e^{z}-1\right)\right], y \geq 1$ and $z \in(-1,1)$,

$$
g(y+w)-\theta(y+w) \geq g(y)-\theta y e^{z} \geq A y-\theta(e-1) y>0 .
$$

Hence,

$$
\begin{aligned}
\mathbb{E}_{x}\left[\int_{0}^{t \wedge T_{M}} \int_{(0,1)}\left(G\left(e^{z} Z_{s}\right)-G\left(Z_{s}\right)-\frac{\left(e^{z}-1\right) Z_{s}}{g\left(Z_{s}\right)-\theta Z_{s}}\right) \pi(\mathrm{d} z) \mathrm{d} s\right] \\
\leq \mathbb{E}_{x}\left[\int_{0}^{t \wedge T_{M}} \int_{(0,1)}\left(\frac{\left(e^{z}-1\right) Z_{s}}{g\left(Z_{s}\right)-\theta Z_{s} e^{z}}-\frac{\left(e^{z}-1\right) Z_{s}}{g\left(Z_{s}\right)-\theta Z_{s}}\right) \pi(\mathrm{d} z) \mathrm{d} s\right] \\
\leq \mathbb{E}_{x}\left[\int_{0}^{t \wedge T_{M}} \int_{(0,1)} \frac{\theta\left(e^{z}-1\right)^{2}\left(Z_{s}\right)^{2}}{\left(A Z_{s}-\theta\left(e^{1}-1\right) Z_{s}\right)\left(g\left(Z_{s}\right)-\theta Z_{s}\right)} \pi(\mathrm{d} z) \mathrm{d} s\right] \\
\leq \mathbb{E}_{x}\left[t \wedge T_{M}\right] \frac{\theta \int_{(0,1)} z^{2} \pi(\mathrm{d} z)}{A\left(A-\theta\left(e^{1}-1\right)\right)} .
\end{aligned}
$$


Similarly, we deal with the second part of the integral and deduce

$$
\mathbb{E}_{x}\left[\int_{0}^{t \wedge T_{M}} \int_{(-1,0)}\left(G\left(e^{z} Z_{s}\right)-G\left(Z_{s}\right)-\frac{\left(e^{z}-1\right) Z_{s}}{g\left(Z_{s}\right)-\theta Z_{s}}\right) \pi(\mathrm{d} z) \mathrm{d} s\right] \leq \mathbb{E}_{x}\left[t \wedge T_{M}\right] \frac{\theta \int_{(-1,0)} z^{2} \pi(\mathrm{d} z)}{A^{2}} .
$$

Thus putting all pieces together (i.e. inequalities (2.9), (2.10), (2.11), (2.12), (2.13) and the bound found in (5) together with (2.8), (2.6) and the three null-expectations), we deduce

$$
\mathbb{E}_{x}\left[\int_{x}^{Z_{t \wedge T_{M}}} \frac{\mathrm{d} w}{g(w)-\theta w}\right] \leq-C(A) \mathbb{E}\left[t \wedge T_{M}\right],
$$

with $C(A)>0$. In other words, for any $x, t \geq 0$,

$$
\mathbb{E}_{x}\left[t \wedge T_{M}\right] \leq \frac{1}{C(A)} \mathbb{E}_{x}\left[\int_{Z_{t \wedge T_{M}}}^{x} \frac{\mathrm{d} w}{g(w)-\theta w}\right] \leq \frac{1}{C(A)} \int_{M e^{-1}}^{\infty} \frac{\mathrm{d} w}{g(w)-\theta w}
$$

Hence using the Monotone Convergence Theorem, as $t$ goes to $\infty$, we deduce (2.3).

In order to prove that the process becomes extinct almost surely, we first show that the time to extinction for the process $Z$ starting from $M$ is not almost surely infinite. We recall that we assumed that the environment has no negative jumps larger than $1-e^{-1}$. Using Proposition 1.1 (both processes with the same restriction on the negative jumps of the environment), we observe that for any $x \leq M$, the process $\left(Z, \mathbb{P}_{x}\right)$ is stochastically dominated by $\left(Z^{\sharp}, \mathbb{P}_{x}^{\sharp}\right)$. The process $Z^{\sharp}$ is a CB-process in a Lévy random environment (without competition) which is characterized by the branching mechanism $\psi(\lambda)$. Since $\psi$ satisfies Grey's condition, Theorem 4.1 of [14] ensures that there is $t_{0}>0$ for which

$$
0<\mathbb{P}_{M}^{\sharp}\left(Z_{t_{0}}^{\sharp}=0\right) \leq \inf _{x \leq M} \mathbb{P}_{x}\left(Z_{t_{0}}=0\right):=p .
$$

Then we denote by $T_{M}^{x}$ for the stopping time $T_{M}$ under $\mathbb{P}_{x}$. Reasoning by recurrence and using the Markov property, we prove that the extinction time of $Z$ is stochastically dominated from above by the random variable $\sum_{i=1}^{\xi}\left(\bar{\tau}_{i}+t_{0}\right)$, where $\xi$ is a geometric random variable that counts the number of random steps before $Z$ becomes extinct and $\left\{\bar{\tau}_{i}\right\}_{i \geq 0}$ are i.i.d., independent of $\xi$ and have the same distribution as $\sup _{x \geq 0} T_{M}^{x}$. To be more precise, the algorithm is as follows: we start from $x$, we wait a random time $\tau_{1} \leq \bar{\tau}_{1}$ until the process is below the level $M$ and then the process becomes extinct before an time interval of size $t_{0}$ with probability $p$. If the process is not extinct after the time $\tau_{1}+t_{0}$, we start again the procedure thanks to the Markov property. Hence,

$$
\sup _{x \geq 0} \mathbb{E}_{x}\left[T_{0}\right] \leq \frac{1}{p}\left(t_{0}+\mathbb{E}\left[\sup _{x \geq 0} T_{M}^{x}\right]\right)=\frac{1}{p}\left(t_{0}+\sup _{x \geq 0} \mathbb{E}_{x}\left[T_{M}\right]\right)<\infty,
$$

and in particular the process becomes extinct a.s.

It remains to prove that the point $\infty$ is a continuous entrance point and that the process can be extended to a Feller process on $[0, \infty]$. Proposition 1.1 guarantees that the sequence of random variables $\left(T_{m}, \mathbb{P}_{x}\right)$ is increasing with respect to $x$. Thus, it converges almost surely to a random variable here denoted by $T_{m}^{\infty}$. Then, from the first part of this proof, for any $m \geq M$,

$$
\sup _{x \geq m} \mathbb{E}_{x}\left[T_{m}\right] \leq \int_{m e^{-1}}^{\infty} \frac{\mathrm{d} w}{g(w)-\theta w} \underset{m \rightarrow \infty}{\longrightarrow} 0 .
$$

From Chebyshev's inequality and the Monotone Convergence Theorem, we deduce that for any $t>0$

$$
\lim _{m \rightarrow \infty} \lim _{x \rightarrow \infty} \mathbb{P}_{x}\left(T_{m}>t\right) \leq \lim _{m \rightarrow \infty} \lim _{x \rightarrow \infty} \frac{E_{x}\left[T_{m}\right]}{t}=0 .
$$


In other words the point $\infty$ is a continuous entrance point.

Next, we prove the extension to a Feller process on $[0, \infty]$. Let $\mathcal{C}_{0}([0, \infty])$ be the set of continuous functions on $[0, \infty]$ that vanish at $\infty$ and recall that $\left(\mathrm{P}_{t}, t \geq 0\right)$ denotes the semigroup associated to the process $Z$ which is Feller on $[0, \infty)$.

Let $t>0$ be fixed. Recall, from Proposition 1.1, that for any non-decreasing sequence $\left\{x_{n}\right\}_{n \geq 1}$ of strictly positive real numbers the sequence of random variable $\left\{Z_{t}, \mathbb{P}_{x_{n}}\right\}_{n \geq 1}$ is non-decreasing. Hence it converges a.s. to a limit that we denote by $Z_{t}^{\infty} \in[0, \infty]$. Then, for any $f \in \mathcal{C}_{0}([0, \infty]), f$ is bounded and from the Dominated Convergence Theorem, we deduce

$$
\mathrm{P}_{t} f(x)=\mathbb{E}_{x}\left[f\left(Z_{t}\right)\right] \underset{x \rightarrow \infty}{\longrightarrow} \mathbb{E}\left[f\left(Z_{t}^{\infty}\right)\right] .
$$

We denote this limit by $\mathrm{P}_{t} f(\infty)$. Let us prove that the extension, defined as previously on $[0, \infty]$, gives a Feller semigroup. The definition through a limit guarantees that $\mathrm{P}$ remains a semigroup on $[0, \infty]$. Thus, according to Chapter III of [29], it is sufficient to prove that for any $f \in \mathcal{C}_{0}([0, \infty])$,

$$
\lim _{t \rightarrow 0}\left\|\mathrm{P}_{t} f-f\right\|_{[0, \infty]}=0 .
$$

Observe that for any $t \geq 0, f \in \mathcal{C}_{0}([0, \infty])$, and $x>0$, we have

$$
\begin{aligned}
\left\|\mathrm{P}_{t} f-f\right\|_{[0, \infty]} & \leq\left\|\mathrm{P}_{t} f-f\right\|_{[0, \infty)}+\left|\mathrm{P}_{t} f(\infty)\right| \\
& \leq 2\left\|\mathrm{P}_{t} f-f\right\|_{[0, \infty)}+\left|\mathrm{P}_{t} f(\infty)-\mathrm{P}_{t} f(x)\right|+|f(x)| .
\end{aligned}
$$

Since $\mathrm{P}$ is a Feller semigroup on $[0, \infty)$, we conclude that the term of the right hand side of the previous inequality is small as soon as $t$ is sufficiently small and $x$ is chosen sufficiently large.

This ends the proof of Theorem 1.2.

Acknowledgements. Both authors acknowledge support from the Royal Society and CONACyTMEXICO. This work was concluded whilst JCP was on sabbatical leave holding a David Parkin Visiting Professorship at the University of Bath, he gratefully acknowledges the kind hospitality of the Department and University

\section{References}

[1] V. I. Afanasyev, C. Böinghoff, G. Kersting, and V. A. Vatutin: Limit theorems for weakly subcritical branching processes in random environment. J. Theor. Probab., 25, 703-732, (2012).

[2] BA, M. And Pardoux, E. Branching processes with interaction and a generalized Ray-Knight theorem. Ann. Inst. Henri Poincaré Probab. Stat., 51, no. 4, 1290-1313, (2015).

[3] V. Bansaye and F. Simatos: On the scaling limits of Galton Watson processes in varying environment. Electron. J. Probab., 20, no. 75, 1-36, (2015).

[4] V. Bansaye : Approximation of stochastic processes by non-expansive flows and coming down from infinity. To appear in Annals in Applied Probability, (2019).

[5] Berestycki, J., Fittipaldi, M.C. and Fontbona, J. Ray-Knight representation of flows of branching processes with competition by pruning of Lévy trees, Probab. Theory Related Fields, 172, 725-788, (2018).

[6] C. Böinghoff, E. E. Dyakonova, G. Kersting, and V. A. Vatutin: Branching processes in random environment which extinct at a given moment, Markov Process. Related Fields 16, 329-350, (2010). 
[7] M.E. Caballero, A. Lambert and G. Uribe Bravo: Proof(s) of the Lamperti representation of continuous-state branching processes. Probab. Surv., 6, 62-89, (2009).

[8] Dawson, D.A. And Li, Z. Stochastic equations, flows and measure-valued processes. Ann. Probab. 40, 813-857, (2012).

[9] Döring, L. And Kyrpianou, A. Entrance and exit at infinity for stable jump diffusions, Preprint, (2018). Available at arXiv: 1802.01672.

[10] El Karoui, N. And MÉlÉArd, S. Martingale measures and stochastic calculus. Probab. Theory Relat. Fields, 84, 83-101, (1990).

[11] Foucart, C. Continuous state branching processes with competition, duality and reflection at infinity Preprint (2017) arXiv:1711.06827.

[12] González-Casanova, A., Pardo, J.C. and Perez., J.L. Branching processes with interactions: the subcritical cooperative regime. Preprint, (2017). arXiv:1704.04203.

[13] A. Grimvall: On the convergence of sequences of branching processes. Ann. Probab., 2, 1027-1045 (1974).

[14] He, H. AND Li, Z. AND Xu, W. Continuous-state branching processes in Lévy random environments J. Theor. Probab., 31, 1952-1974, (2018).

[15] Kallenberg, O. Foundations of Modern Probability. Probability and Its Applications, SpringerVerlag, New York, (1997).

[16] N. KeIding: Extinction and exponential growth in random environments. Theor. Population Biology. 8, 49-63, (1975).

[17] T. Kurtz: Diffusion approximations for branching processes. Branching processes. Adv. Probab. Related Topics, 5, Dekker, New York, 269-292, (1976).

[18] Lambert, A. The branching process with logistic growth, Ann. Appl. Probab., 15, no. 2, 1506-1535, (2005).

[19] LE, V. Processus de branchement avec interaction. PhD Thesis, Univ. Aix-Marseille (2014).

[20] Le, V. And Pardoux, E. Height and the total mass of the forest of genealogical trees of a large population with general competition. ESAIM: Probability and Statistics, 19, 172-193 (2015).

[21] Leman, H. And Pardo, J.C. Extinction time of a CB-processes with competition in a Lévy random environment. arXiv:1801.04501 (2018).

[22] Leman, H. And Pardo, J.C. Extinction time of the logistic branching processes in a Brownian environment. Work in progress (2018).

[23] LI, P.S. A continuous-state polynomial branching process, To appear in Stochastic Processes and their Applications, (2019). Available at arXiv:1609.09593.

[24] Li, P.S., YAng, Z. And Zhou, X. A general continuous-state nonlinear branching process, To appear in Annals of Applied Probability, (2019). Available at arXiv:1708.01560.

[25] MA, R. Lamperti transformation for continuous-state branching processes with competition and applications, Statist. Probab. Lett. 107, 11-17, (2015). 
[26] Palau, S. and Pardo, J.C. Continuous state branching processes in random environment: the Brownian case. Stochastic Process. Appl. 127, no.3, 957-994, (2017).

[27] Palau, S. And Pardo, J.C. Branching processes in a Lévy random environment. Acta Appl. Math., 153, no.1, 55-79, (2018).

[28] Pardoux, E. Probabilistic models of population evolution. Scaling limits, genealogies and interactions. Mathematical Biosciences Institute Lecture Series. Stochastics in Biological Systems, 1.6. Springer ; MBI Mathematical Biosciences Institute, Ohio State University, Columbus, OH, (2016).

[29] Revuz, D. And Yor, M. Continuous martingales and Brownian motion. Third edition, Springer Science \& Business Media, (2005).

[30] W.L. Smith and W.E. Wilkinson: On branching processes in random environments. Ann. Math. Statist., 40, 814-827, (1969). 